\title{
Dynamic Control of ERG20 and ERG9 Expression for Improved Casbene Production in Saccharomyces cerevisiae
}

\author{
Roberta Callari, Yvan Meier, Davide Ravasio and Harald Heider* \\ Evolva SA, Reinach, Switzerland
}

Production of plant metabolites in microbial hosts represents a promising alternative to traditional chemical-based methods. Diterpenoids are compounds with interesting applications as pharmaceuticals, fragrances and biomaterials. Casbene, in particular, serves as a precursor to many complex diterpenoids found in plants from the Euphorbiaceae family that have shown potential therapeutic effects. Here, we engineered the budding yeast Saccharomyces cerevisiae for improved biosynthesis of the diterpene casbene. We first expressed, in yeast, a geranylgeranyl diphosphate synthase from Phomopsys amygdali in order to boost the geranylgeranyl diphosphate pool inside the cells. The enzyme uses isopentenyl diphosphate and dimethylallyl diphosphate to directly generate geranylgeranyl diphosphate. When co-expressing a casbene synthase from Ricinus communis the yeast was able to produce casbene in the order of $30 \mathrm{mg} / \mathrm{L}$. Redirecting the flux from FPP and sterols, by means of the ergosterol sensitive promoter of ERG1, allowed for plasmid-based casbene production of $81.4 \mathrm{mg} / \mathrm{L}$. Integration of the target genes into the yeast genome, together with the replacement of the promoter regions of ERG20 and ERG9 with combinations of ergosterol- and glucose-sensitive promoters, generated a titer of $108.5 \mathrm{mg} / \mathrm{L}$ of casbene. We here succeeded to engineer an improved route for geranylgeranyl diphosphate synthesis in yeast. Furthermore, we showed that the concurrent dynamic control of ERG20 and ERG9 expression, using ergosterol and carbon source regulation mechanisms, could substantially improve diterpene titer. Our approach will pave the way for a more sustainable production of GGPP- and casbene-derived products.

Keywords: casbene, diterpene, ERG20, ERG9, yeast, metabolic engineering, dynamic control, mevalonate pathway

Received: 18 July 2018 Accepted: 16 October 2018 Published: 01 November 2018

Citation:

Callari R, Meier Y, Ravasio D and Heider H (2018) Dynamic Control of ERG20 and ERG9 Expression for Improved Casbene Production in

Saccharomyces cerevisiae.

Front. Bioeng. Biotechnol. 6:160.

doi: 10.3389/fbioe.2018.00160

\section{INTRODUCTION}

Diterpenoids represent one of the largest and most diverse classes of plant metabolites. Although in some cases they carry out important primary functions (e.g., regulation of growth and development by gibberellins), they are usually products of the secondary metabolism with specialized pathways extremely varied across the plant kingdom (Zerbe and Bohlmann, 2015). Diterpenoids are beneficial for plants because of their role in protection from abiotic stress and control of the ecological interactions with other organisms (e.g., defense against herbivores and microbial 
pathogens) (Cheng et al., 2007; Tholl, 2015). Moreover, they can benefit humanity because of their industrial application as pharmaceuticals (e.g., paclitaxel, forskolin, ingenol-3-angelate, prostratin), fragrances (e.g., sclareol), and other industrial bioproducts (e.g., steviol glycosides as natural sweetners, diterpene resins for inks, and coatings) (Bohlmann and Keeling, 2008; Goyal et al., 2010; Caniard et al., 2012; Doseyici et al., 2014; Fidler and Goldberg, 2014; Howat et al., 2014; Miana et al., 2015).

Current production methods of diterpenoids rely on extraction from natural sources and chemical synthesis. Because of the low yield occurrence in the producing organisms and the structural complexity of the compounds, such methods are inefficient and environmentally costly. An attractive and environmentally friendly alternative is represented by microbial fermentation. Biosynthesis in heterologous hosts such as Escherichia coli or Saccharomyces cerevisiae can (1) reduce costs using sugar-based carbon sources, (2) increase sustainability by avoiding harvesting and extraction from natural sources, (3) increase yield and productivity using genetic manipulation of the heterologous hosts, and (4) provide enantiomerically pure products through enzymatic biocatalysis (Scalcinati et al., 2012). S. cerevisiae in particular is a robust host that not only offers the biosynthetic machinery needed for production of diterpenoids, but also contributes the necessary environment for expression of membrane-bound enzymes, such as cytochrome P450 hydroxylases. These p450 enzymes are frequently involved in the biosynthesis of complex plant terpenoids and are usually difficult to express in prokaryotic systems (Hamann and Møller, 2007; Kirby and Keasling, 2009).

The precursors for production of terpenes are present in the native metabolic network of $S$. cerevisiae (Chambon et al., 1991). The yeast mevalonate (MVA) pathway, through multiple rounds of condensation of isopentenyl diphosphate (IPP) and dimethylallyl diphosphate (DMAPP), leads to generation of geranyl diphosphate (GPP), farnesyl diphosphate (FPP) and geranylgeranyl diphosphate (GGPP) (Figure 1). GPP, FPP, and GGPP represent the universal precursor units of all monoterpenes $\left(\mathrm{C}_{10}\right)$, sesquiterpenes $\left(\mathrm{C}_{15}\right)$, and diterpenes $\left(\mathrm{C}_{20}\right)$ respectively. However, the GGPP content in S. cerevisiae is rather low, due to the fact that the endogenous geranylgeranyl diphosphate synthase (GGPPS) Bts1p does not compete efficiently with enzymes directly upstream and downstream (farnesyl diphosphate synthase Erg20p and squalene synthase Erg9p) for the pools of IPP and FPP (Jiang et al., 1995). Thus, the endogenous flux of the pathway favors production of FPP, and the sterols derived from it. As a consequence, improvement of the GGPP pool is essential for efficient, hightiter diterpene production. To reach this goal different studies have focused on either the use of heterologous GGPP synthases, Erg20p mutants that are able to synthetize GGPP, or on fusions

\footnotetext{
Abbreviations: MVA, mevalonate; IPP, isopentenyl diphosphate; DMAPP, dimethylallyl diphosphate; GPP, geranyl diphosphate; FPP, farnesyl diphosphate; GGPP, geranylgeranyl diphosphate; GGPPS, geranylgeranyl diphosphate synthase; PaGGPPS, geranylgeranyl diphosphate synthase from Phomopsis amygdali; RcCBS, casbene synthase from Ricinus communis; CAS, casbene; $\mathrm{OD}_{600}$, optical density at $600 \mathrm{~nm}$; PaFS, fusicoccadiene synthase from Phomopsis amygdali; ORF, open reading frame.
}

of BTS1 and ERG20 (Kirby et al., 2010; Zhou et al., 2012; Ignea et al., 2015; Song et al., 2017). Such strategies have been shown to improve production of GGPP-derived compounds, but they have also demonstrated that additional improvements in substrate conversion toward diterpene production are feasible.

In this study, we developed a yeast platform for production of the diterpene hydrocarbon casbene, originally identified from castor bean (Ricinus communis) where it serves as a phytoalexin (Mau and West, 1994). In the last decades, the compound has gained much interest since the conversion of GGPP into casbene, catalyzed by the casbene synthase enzyme, is acknowledged as the first committed step in the biosynthesis of the diterpenoid molecular backbones jatrophane, tigliane, lathyrane, and ingenane (Kirby et al., 2010). Casbene is the precursor of many diterpenoids of medical interest, identified in a number of Euphorbiaceae (Figure 2). For example, Ingenol3-angelate, found in the sap of Euphorbia peplus, has been approved by the FDA for the treatment of actinic keratosis (Fidler and Goldberg, 2014) whilst Prostratin, derived from Homolanthus nutans is a protein kinase $\mathrm{C}$ activator that inhibits HIV-1 infections and reduces HIV-1 latency (Miana et al., 2015). Jatrophone, isolated from extracts of Jatropha gossypifolia L., has shown significant anti-proliferative effects against human tumor cell lines (Kupchan et al., 1970; Theoduloz et al., 2009) and the tigliane derived diterpenoid, resiniferatoxin from Euphorbia resinifera is effective against a broad range of inflammatory and neuropathic pain conditions (Iadarola and Gonnella, 2013). Euphorbia factor L2 from Euphorbia lathyris induces apoptosis in lung cancer cell lines (Lin et al., 2017).

For efficient production of casbene in yeast, we increased the GGPP supply inside the cells by expression of a truncated, fungal GGPP synthase, from the endophytic fungus Phomopsis amygdali. Expression of the enzyme, producing GGPP from IPP and DMAPP directly, significantly improved GGPP biosynthesis and allowed, upon co-expression of the casbene synthase from $R$. communis, for production of casbene in the order of 30 $\mathrm{mg} / \mathrm{L}$. Similar titers have been reported previously (Kirby et al., 2010). Dynamic control of genes ERG20 and ERG9 by means of ergosterol- and glucose-sensitive promoters further redirected the flux to GGPP and diterpene synthesis, improving casbene production by up to $108.5 \mathrm{mg} / \mathrm{L}$.

We envision that our approach will pave the way toward the sustainable production of various GGPP- and casbene-derived isoprenoids.

\section{MATERIALS AND METHODS}

\section{Chemicals and Media}

All chemicals were bought from Sigma-Aldrich (St. Louis Missouri, USA) unless stated otherwise. Authentic standard of cembrene was purchased from CHEMOS GmbH \& Co. KG (Regenstauf, Germany). Authentic standard of casbene was received from Professor Birger Møller's laboratory at the University of Copenhagen.

LB medium for growth of Escherichia coli was supplied from Carl Roth GmbH + Co. KG (Karlsruhe, Germany), and was 


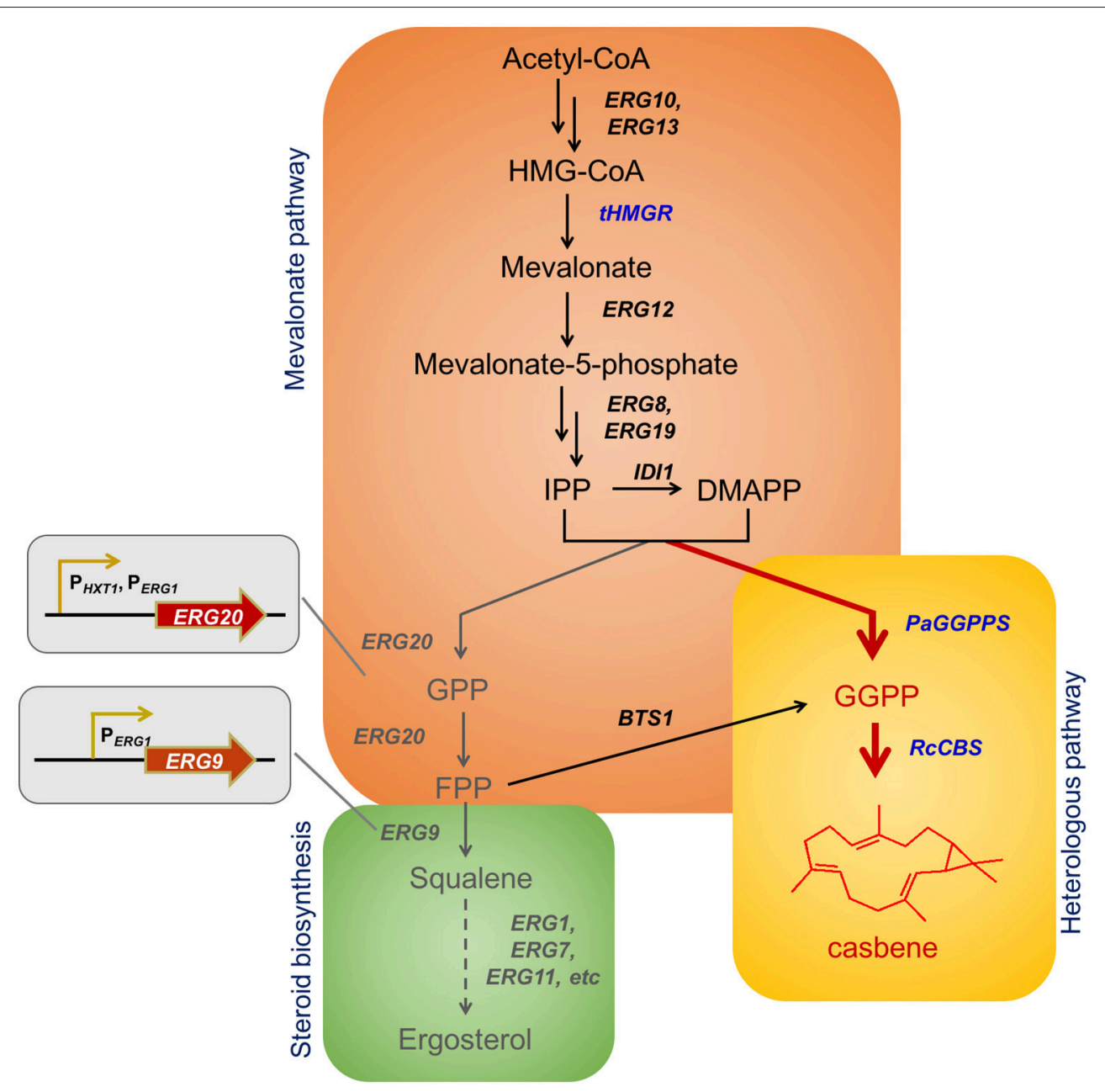

FIGURE 1 | Biosynthesis of casbene in yeast and examples of casbene derived compounds. Schematic overview of casbene biosynthesis based on engineered mevalonate pathway in S. cerevisiae. The new biosynthetic branch starts with PaGGPPS, for production of GGPP using IPP and DMAPP as sole substrates. RcCBS mediates casbene synthesis (in red). Endogenous genes and metabolites are shown in black. Overexpressed genes are shown in blue. Genes regulated through dynamic control (ERG20 and ERG9) and genes from the steroid biosynthetic pathway are shown in gray. The truncated endogenous gene tHMG1 and the heterologous genes PaGGPPS (encoding a truncated version of the fusicoccadiene synthase from Phomopsis amygdali serving as GGPP synthase) and RcCBS (encoding a truncated version of the casbene synthase from Ricinus communis) were overexpressed to improve casbene production. The native promoter of ERG20 was replaced with $\mathrm{P}_{E R G 1}$ or $\mathrm{P}_{H X T 1}$, respectively. The native promoter of ERG9 was replaced with $\mathrm{P}_{E R G 1}$. HMG-CoA, 3-hydroxy-3-methylglutaryl coenzyme A; IPP, isopentenyl pyrophosphate; DMAPP, dimethylallyl pyrophosphate; GPP, geranyl diphosphate; FPP, farnesyl diphosphate; GGPP, geranylgeranyl diphosphate.

supplemented with $100 \mu \mathrm{g} / \mathrm{L}$ of ampicillin for amplification of plasmids. Yeast Extract Peptone Dextrose (YPD) medium with $20 \mathrm{~g} / \mathrm{L}$ glucose was used for growth of wildtype strains prior to transformation. For preparation of pre- and main cultures we used synthetic complete (SC) drop-out medium (Formedium LTD, Hustanton, England), supplied with $6.7 \mathrm{~g} / \mathrm{L}$ yeast nitrogen base, $20 \mathrm{~g} / \mathrm{L}$ glucose and all amino acids necessary for the corresponding auxotrophy.

\section{Plasmids and Strains Construction}

Table 1 lists all plasmids constructed in this work. Coding sequences (Table S1) were synthesized by GeneArt ${ }^{\circledR}$ (Thermofisher Scientific, Zug, Switzerland) as yeast codon optimized versions. Synthetic genes were cloned via HindIII HF, SacII restriction digestion and T4 DNA ligase (New England
Biolabs, Ipswich, Massachusetts, USA) according to standard protocols (Green and Sambrook, 2012). E. coli XL10 Gold (Agilent, Santa Clara, California, USA) cells were used for subcloning of genes.

S. cerevisiae strains generated throughout this study are listed in Table 2. All constructed strains were derived from strain NCYC 3608 (NCYC, Norwich, United Kingdom), a derivative of strain S288C modified in our labs and carrying a truncated 3-hydroxy-3-methylglutaryl coenzyme A reductase (tHMGR) in the YCT1 locus to increase the flow through the early part of the mevalonate pathway (CAS1- MATalpha ho $\Delta 0$ his3 $\Delta 0$ leu2 $\Delta 0$ ura3 $\Delta 0$ CAT5(T91M) MIP1(-661T) SAL1 GAL2 YLL055w::loxPpTDH3-Sc_tHMGR-tCYC1). All yeast strains were stored in 25\% glycerol at $-80^{\circ} \mathrm{C}$. Yeast transformation was performed using the classical lithium acetate method (Gietz and Schiestl, 2007). 


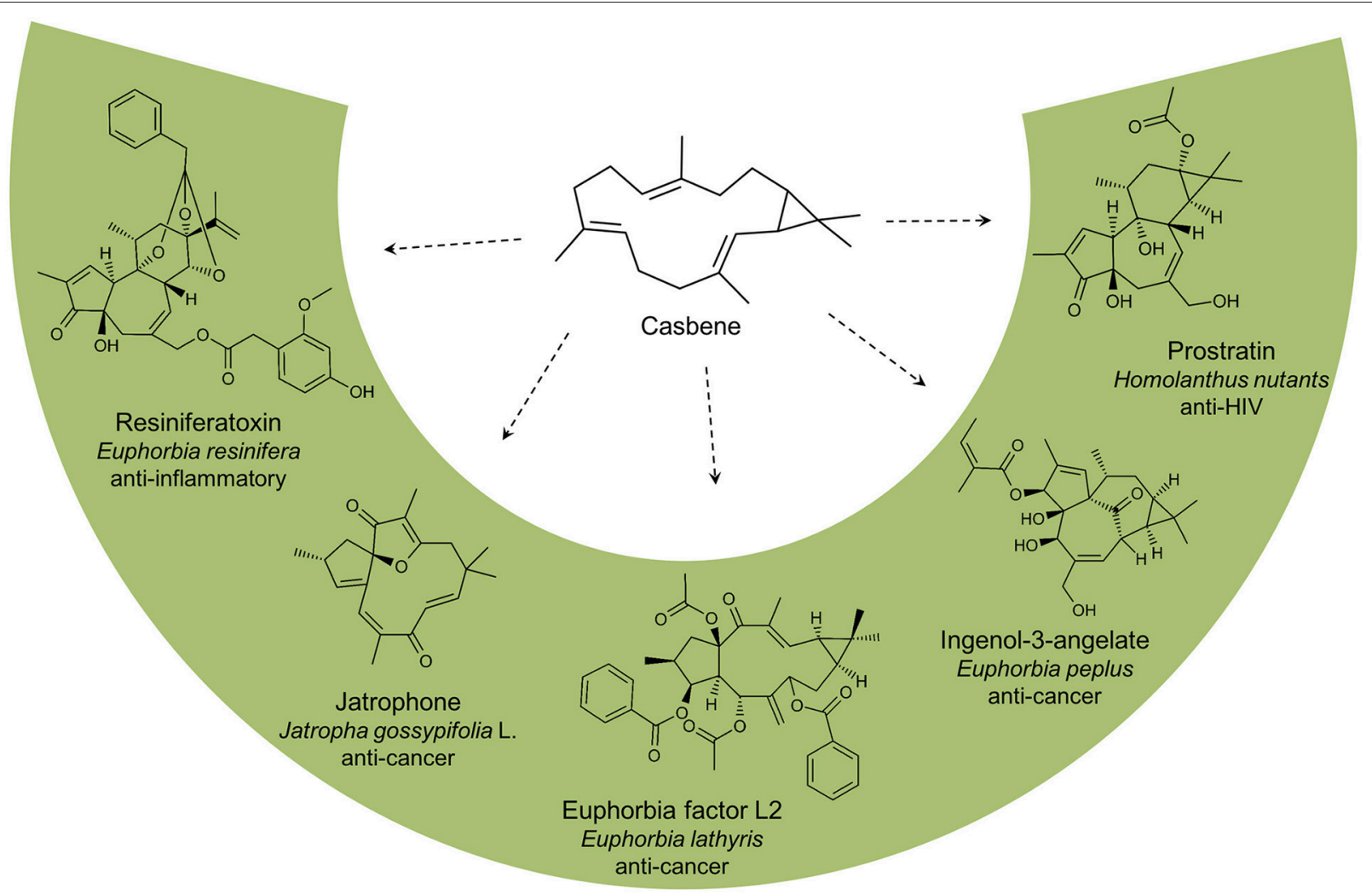

FIGURE 2 | Casbene is the precursor of many diterpenoids identified in plants from the Euphorbiaceae family. Euphorbia factor L2 (Euphorbia lathyris), resiniferatoxin (Euphorbia resinifera), ingenol-3-angelate (Euphorbia peplus), prostratin (Homolanthus nutans), and jatrophone (Jatropha gossypifolia L.).

Transformants were grown on agar plates prepared with selective SC drop-out medium. For integration into the yeast genome, genes were cloned into yeast integration plasmids targeting incorporation into specific sites (Table 1). Transformants were verified by PCR on genomic DNA for correct insertion of heterologous genes.

The 805 bp promoter region of ERG1 (Table S2) was amplified from genomic DNA of $S$. cerevisiae S288C after lysis in 30 $\mu \mathrm{L} 0.2 \% \mathrm{SDS}$ at $95^{\circ} \mathrm{C}$ for $5 \mathrm{~min}$ and clarification at $14,000 \mathrm{~g}$ for $5 \mathrm{~min}$. iProof $^{\mathrm{TM}}$ High-Fidelity DNA Polymerase was used according to the manufacturer's protocol with primer pair F_ERG1p_SpeI/ R_ERG1p_SacII. The PCR product was cut with SpeI/SacII, and inserted into the integration vector pUG72 (Gueldener et al., 2002) close to the URA3 marker flanked by loxP sites. Marker and promoter were amplified with primers pair F_ERG9int/ R_ERG9int and transformed in yeast for homologous recombination at the ERG9 site (Table 3).

Plasmid pCAS4 for replacement of the promoter region of ERG20 (Table S2) was kindly provided by Michael Eichenberger. The plasmid already contained up- and down-tags for homologous recombination at the ERG20 site, a URA3 marker and the promoter of $\mathrm{CYC1}$ for exchange with the promoter of ERG20. $\mathrm{P}_{C Y C 1}$ was released from the plasmid with BglII and HindIII and replaced by promoters of ERG1 and HXT1. $\mathrm{P}_{E R G 1}$ was amplified from plasmid pCAS3 with primers pair F_ERG1p_BglII and R_ERG1p_HindIII. $\mathrm{P}_{H X T 1}$ was amplified from an expression vector found in our labs with primers pair F_HXT1p_BglII and R_HXT1p_HindIII (Table 3). The PCR products were cut with BglII/HindIII, and inserted into the integration vector pCAS3 to replace $\mathrm{P}_{C Y C 1}$. Strains with successful replacement of native promoters of ERG20 and ERG9 were verified by diagnostic PCR.

\section{Yeast Growth}

Yeast batch cultures for production of metabolites were performed with the System Duetz (EnzyScreen, Heemstede, Netherlands) in an ISF-1-W shaker (Kuhner, Birsfelden, Switzerland) at $30^{\circ} \mathrm{C}, 300 \mathrm{rpm}$, and $5 \mathrm{~cm}$ shaking diameter. Precultures were prepared by inoculation of single colonies into $3 \mathrm{~mL}$ of selective liquid SC medium in triplicates. Pre-cultures were grown for $24 \mathrm{~h}$ at $30^{\circ} \mathrm{C}, 160 \mathrm{rpm}$. Optical density at $600 \mathrm{~nm}$ $\left(\mathrm{OD}_{600}\right)$ of a 1:40 dilution was measured in an Ultrospec 10 table top spectrophotometer (GE Healthcare, Little Chalfont, United Kingdom). Main cultures were inoculated in $2.5 \mathrm{~mL}$ of selective SC medium in a 24-deepwell microplate to an $\mathrm{OD}_{600}$ of 0.1 and incubated from 24 up to $120 \mathrm{~h}$ as stated.

\section{Sample Preparation}

Cells from a $2 \mathrm{~mL}$ volume were harvested by centrifugation at 4,000 rpm for $5 \mathrm{~min}$ in $2 \mathrm{~mL}$ screw cap tubes. Supernatants were discarded and pellets were mixed with $500 \mu \mathrm{L}$ of methanol. Pellets were shaken for $10 \mathrm{~min}$ at $60^{\circ} \mathrm{C}$ and $1,500 \mathrm{rpm}$ in a Thermo-Shaker TS-100 (Axonlab, Reichenbach an der Fils, Germany). Cell debris was removed by centrifugation at 4,000 
rpm for $5 \mathrm{~min}$ and liquid phases were transferred to glass vials. The methanol extracts were evaporated for $24 \mathrm{~h}$ at room temperature under the fume hood, resolubilized in $500 \mu \mathrm{L}$ hexane and used for analysis.

\section{Detection of Diterpenes}

Casbene and alcohols derived from FPP and GGPP were detected using gas chromatography-mass spectrometry (GC-MS). Quantification was carried out using the Agilent Technologies 7890a GC system (Agilent Technologies, Santa Clara, USA) equipped with a 5975 Mass Selective Detector (MSD). Sample volumes of $1 \mu \mathrm{L}$ were injected in splitless mode at $250^{\circ} \mathrm{C}$ with the following GC program: $80^{\circ} \mathrm{C}$ for $2 \mathrm{~min}$, raise to $220^{\circ} \mathrm{C}$ at $25^{\circ} \mathrm{C} / \mathrm{min}$, raise to $240^{\circ} \mathrm{C}$ at $3^{\circ} \mathrm{C} / \mathrm{min}$, ramp at rate $80^{\circ} \mathrm{C} / \mathrm{min}$ to $300^{\circ} \mathrm{C}$ and held for $3 \mathrm{~min}$. Helium was used as carrier gas with a constant flow rate of $1.2 \mathrm{~mL} / \mathrm{min}$. The MSD was operated at $70 \mathrm{eV}$ in scan mode, with a range between 35 and $500 \mathrm{~m} / z$. Diterpenes were identified by retention time and comparison with a mass spectral database (NIST version 2.0, Gaithersburg, MD, USA) and/or comparison with authentic standards. $\beta$-Caryophyllene $(10 \mathrm{mg} / \mathrm{L})$ was added in each sample as internal standard and calibration curves were generated to quantify the target compounds. In the case of geranylgeranyol an authentic standard of the compound was used, whereas casbene quantification was approximated by a standard curve generated using an authentic standard of the diterpene cembrene.

\section{RESULTS}

\section{Establishing Casbene Production in S. cerevisiae}

In order to assess casbene (CAS) production in yeast, the casbene synthase gene from $R$. communis ( $R c C B S$ ) was cloned as a codon optimized version under control of the promoter of TEF1 in a yeast expression plasmid ("pCAS1"). The N-terminal

TABLE 1 | List of plasmids constructed in this work.

\begin{tabular}{|c|c|c|}
\hline Plasmid & Description & Plasmid type \\
\hline pCAS1 & $\begin{array}{l}\text { Plasmid harboring expression cassette } \\
\text { pTEF1-RcCBS-ENO2t }\end{array}$ & ARS/CEN \\
\hline pCAS2 & $\begin{array}{l}\text { Plasmid harboring expression cassette } \\
\text { pPGK1-PaGGPPS-ADH2t }\end{array}$ & ARS/CEN \\
\hline pCAS3 & $\begin{array}{l}\text { Plasmid for ERG9 promoter exchange with } \\
\text { insertion of the promoter region from ERG1 } \\
\text { gene }\end{array}$ & Integrative \\
\hline pCAS4 & $\begin{array}{l}\text { Plasmid for ERG20 promoter exchange with } \\
\text { insertion of the promoter region from CYC1 } \\
\text { gene }\end{array}$ & Integrative \\
\hline pCAS5 & $\begin{array}{l}\text { Plasmid for ERG20 promoter exchange with } \\
\text { insertion of the promoter region from ERG1 } \\
\text { gene }\end{array}$ & Integrative \\
\hline pCAS6 & $\begin{array}{l}\text { Plasmid for ERG20 promoter exchange with } \\
\text { insertion of the promoter region from HXT1 } \\
\text { gene }\end{array}$ & Integrative \\
\hline pCAS7 & $\begin{array}{l}\text { Plasmid for pTEF1-RcCBS-tENO2 cassette } \\
\text { integration into YOR092W locus }\end{array}$ & Integrative \\
\hline pCAS8 & $\begin{array}{l}\text { Plasmid for pPGK1-PaGGPPS-tADH2 cassette } \\
\text { integration into YDR122W locus }\end{array}$ & Integrative \\
\hline
\end{tabular}

chloroplast transit peptide, identified with the ChloroP software used at default settings (Emanuelsson et al., 1999) and needed for plastidial localization in plants, was removed (Kirby et al., 2010). Plasmid pCAS1 was introduced into yeast strain H-MEV, engineered for production of isoprenoids. H-MEV contained a chromosomally integrated copy of a truncated 3-hydroxy-3methylglutaryl coenzyme A reductase ( $t H M G R)$ enabling high mevalonate pathway activity (Donald et al., 1997). The resulting strain (CAS1), was analyzed for production of casbene. Small amounts of the compound $(\sim 0.8 \mathrm{mg} / \mathrm{L})$ could be detected after $72 \mathrm{~h}$ of growth (data not shown).

To boost casbene production, we co-expressed a heterologous GGPP synthase from the plant-pathogenic fungus Phomopsis amygdali. Toyomasu et al. (2007) found that synthesis of the diterpene fusicoccadiene in $P$. amygdali is catalyzed by the unusual chimeric enzyme fusioccadiene synthase (PaFS). This multifunctional enzyme can synthetize fusicoccadiene starting from IPP and DMAPP, through (1) a prenyltransferase domain, synthetizing GGPP from the C5 isoprene units, and (2) a

TABLE 2 | List of strains constructed in this work.

\begin{tabular}{|c|c|c|}
\hline Strains & Description & $\begin{array}{l}\text { MVA/sterols pathway } \\
\text { modifications }\end{array}$ \\
\hline H-MEV & $\begin{array}{l}\text { S288C derivative with relatively high } \\
\text { mevalonate pathway activity }\end{array}$ & tHMGR \\
\hline CAS1 & $\begin{array}{l}\text { Strain H-MEV harboring plasmid pCAS1 } \\
\text { and empty vector }\end{array}$ & tHMGR \\
\hline CAS2 & $\begin{array}{l}\text { Strain H-MEV harboring plasmid pCAS1 } \\
\text { and pCAS2 }\end{array}$ & tHMGR \\
\hline CAS3 & $\begin{array}{l}\text { H-MEV with ERG2O under the control of } \\
\text { PERG1 }^{\text {E }}\end{array}$ & tHMGR, $P_{E R G 1}-E R G 20$ \\
\hline CAS4 & $\begin{array}{l}\text { H-MEV with ERG9 under the control of } \\
\mathrm{P}_{E R G 1}\end{array}$ & tHMGR, $P_{E R G 1}-E R G 9$ \\
\hline CAS5 & $\begin{array}{l}\text { H-MEV with ERG2O and ERG9 under the } \\
\text { control of } P_{E R G 1}\end{array}$ & $\begin{array}{l}\text { tHMGR, } \mathrm{P}_{E R G 1}-\text { ERG20, } \\
\mathrm{P}_{\text {ERG1 }}-\text { ERG9 }\end{array}$ \\
\hline CAS6 & $\begin{array}{l}\text { Strain CAS3 harboring plasmid pCAS1 } \\
\text { and pCAS2 }\end{array}$ & tHMGR, $P_{E R G 1}-E R G 20$ \\
\hline CAS7 & $\begin{array}{l}\text { Strain CAS4 harboring plasmid pCAS1 } \\
\text { and pCAS2 }\end{array}$ & tHMGR, $P_{E R G 1}-E R G 9$ \\
\hline CAS8 & $\begin{array}{l}\text { Strain CAS5 harboring plasmid pCAS1 } \\
\text { and pCAS2 }\end{array}$ & $\begin{array}{l}\text { tHMGR, } \mathrm{P}_{E R G 1}-\text { ERG20, } \\
\mathrm{P}_{E R G 1}-\text { ERG9 }\end{array}$ \\
\hline CAS9 & $\begin{array}{l}\text { Strain } \mathrm{H}-\mathrm{MEV} \text { with ERG2O under the } \\
\text { control of } \mathrm{P}_{H T X 1}\end{array}$ & tHMGR, $P_{H X T 1}-E R G 2 O$ \\
\hline CAS10 & $\begin{array}{l}\text { Strain CAS9 with ERG9 under the control } \\
\text { of } \mathrm{P}_{E R G 1}\end{array}$ & $\begin{array}{l}\text { tHMGR, } \mathrm{P}_{H X T 1}-E R G 20, \\
\mathrm{P}_{E R G 1}-\text { ERG9 }\end{array}$ \\
\hline CAS11 & $\begin{array}{l}\text { Strain H-MEV with integrated PaGGPPS } \\
\text { and RcCBS }\end{array}$ & tHMGR \\
\hline CAS12 & $\begin{array}{l}\text { Strain CAS3 with integrated PaGGPPS } \\
\text { and RcCBS }\end{array}$ & tHMGR, $P_{E R G 1}-E R G 20$ \\
\hline CAS13 & $\begin{array}{l}\text { Strain CAS9 with integrated PaGGPPS } \\
\text { and RcCBS }\end{array}$ & tHMGR, $\mathrm{P}_{H X T 1}-$ ERG2O \\
\hline CAS14 & $\begin{array}{l}\text { Strain CAS5 with integrated PaGGPPS } \\
\text { and RcCBS }\end{array}$ & $\begin{array}{l}\text { tHMGR, } \mathrm{P}_{E R G 1}-E R G 20, \\
\mathrm{P}_{E R G 1}-\text { ERG9 }\end{array}$ \\
\hline CAS15 & $\begin{array}{l}\text { Strain CAS10 with integrated PaGGPPS } \\
\text { and RcCBS }\end{array}$ & $\begin{array}{l}\text { tHMGR, } P_{H X T 1}-E R G 20, \\
P_{E R G 1}-E R G 9\end{array}$ \\
\hline
\end{tabular}


terpene cyclase domain, catalyzing the cyclization of GGPP into fusicoccadiene.

We reasoned that the expression of the prenyltransferase domain from PaFS in yeast, catalyzing all steps from the C5 to the C20 precursor unit, could enhance the efficiency of this conversion, as it requires only one enzyme for the entire biosynthesis of GGPP. A construct encoding for the codon optimized prenyltransferase domain (residues 390-719 of PaFS) (Chen et al., 2016) was synthesized and cloned under control of the promoter of PGK1 into pCAS2 yeast expression vector. pCAS2 was introduced together with pCAS1 $(R c C B S)$ into $\mathrm{H}-\mathrm{MEV}$, generating strain CAS2. PaGGPPS + RcCBS co-expression gave rise to a peak with the same retention time and mass-spectrum of the authentic casbene standard (Figure S1A), while, as previously discussed, only trace amounts of the compound accumulated when expressing solely RcCBS (Figure S1B). A titer of $32 \mathrm{mg} / \mathrm{L}$ of casbene was reached in strain CAS2 $(P a G G P P S+R c C B S)$ after $72 \mathrm{~h}$ of batch culture. The relatively high titer of casbene strongly suggested that $P a G G P P S$ could considerably increase the supply of the GGPP precursor necessary for casbene production by RcCBS. This suggestion was further supported by the appearance of a pronounced peak in the chromatogram corresponding to GGOH (Figure S1B), the prenyl alcohol accumulating in engineered yeast cells due to endogenous phosphatase activities acting on the excess amounts of GGPP (Faulkner et al., 1999; Tokuhiro et al., 2009).

\section{Casbene Production in Engineered Strains With Engineered Mevalonate Pathway}

To further improve casbene titers, we focused on downregulation of competing metabolic pathways. PaGGPPSp uses IPP and DMAPP to catalyze the consecutive condensation steps needed for GGPP synthesis and is in competition with the native farnesyl diphosphate synthase, Erg20p for the IPP/DMAPP

TABLE 3 | Oligonucleotides used for plasmid construction (restriction sites are underlined).

\begin{tabular}{|c|c|}
\hline Name & Description \\
\hline F_GGPPS_HindIII & $\begin{array}{l}\text { cgaAAGCTTATGTTGTCTACTGGTTGTCTTा } \\
\text { GTCTCC }\end{array}$ \\
\hline R_GGPPS_Sacll & AAGCACTCCGCGGTTAGTG \\
\hline F_ERG1p_Spel & cgaACTAGTGTCGAATACTACTATGACCGC \\
\hline R_ERG1p_Sacll & acgCCGCGGCATGACCCTITCTCGATATGTT \\
\hline F_ERG9int & $\begin{array}{l}\text { GGTTTGGGTTAGTGCCTAAACGAGCA } \\
\text { GCGAGAACACGACCACGCAGCTGAA } \\
\text { GCTTCGTACGC }\end{array}$ \\
\hline R_ERG9int & $\begin{array}{l}\text { CTTCATCTCGACCGGATGCAATGCCAATT } \\
\text { GTAATAGCTITCCCATACTCAC } \\
\text { TATAGGGAGACCGGC }\end{array}$ \\
\hline F_ERG1p_Bg/ll & ctAGATCTGTCGAATACTACTATGACCGC \\
\hline R_ERG1p_HindIII & ctgAAGCTTATGACCCTITCTCGATATGTT \\
\hline F_HXT1p_Bglll & tcAGATCTCAAGTGCTGATAGAAGAATACCAC \\
\hline R_HXT1p_HindllI & gcgAAGCTTGATTITACGTATATCAACTAGTTGACG \\
\hline
\end{tabular}

pool. Moreover, FPP, produced by Erg20p, enters the ergosterol biosynthetic pathway by action of the squalene synthase Erg9p. Thus, in order to redirect the flux to diterpene production, we attempted dynamic control of either ERG20, or of ERG20 in combination with $E R G 9$, by replacing their native promoters with the ergosterol sensitive promoter $\mathrm{P}_{E R G 1}$. This approach has recently been shown to positively affect amorpha-4-11diene production in yeast (Yuan and Ching, 2015). The promoter of ERG1 senses intracellular ergosterol levels and adjusts transcription of downstream genes to enable production of optimal levels of this essential metabolite. We constructed three strains containing (1) ERG20 under control of $\mathrm{P}_{E R G 1}$, (2) ERG9 under control of $\mathrm{P}_{E R G 1}$ and (3) both ERG20 and ERG9 under control of $\mathrm{P}_{E R G 1}$ (Table 2, CAS3-5). All engineered strains also carried an integrated copy of $t H M G R$ and were transformed with pCAS1 (RcCBS) and pCAS2 (PaGGPPS) to evaluate the effect of dynamic control of expression of ERG20 and/or ERG9 on casbene production. The resulting strains were designated as CAS6 ( $\left.\mathrm{P}_{E R G 1}-E R G 20\right)$, CAS7 $\left(\mathrm{P}_{E R G 1}-E R G 9\right)$ and CAS8 ( $\mathrm{P}_{E R G 1}-E R G 20$ and $\left.\mathrm{P}_{E R G 1}-E R G 9\right)$ (Table 2). CAS2, containing the native promoters of ERG20 and ERG9, was used as a reference strain. Strains were analyzed for casbene production in time course experiments up to $120 \mathrm{~h}$ cultivation time.

Interestingly, strains with engineered ERG20 and ERG9 expression improved the casbene titers substantially but showed different profiles of compound accumulation (Figure 3A). The two strains CAS6 ( $\left.\mathrm{P}_{E R G 1}-E R G 20\right)$ and CAS8 ( $\mathrm{P}_{E R G 1}-E R G 20$, $\left.\mathrm{P}_{E R G 1}-E R G 9\right)$ showed similar profiles of casbene accumulation over time. Strain CAS6 $\left(\mathrm{P}_{E R G 1}-E R G 20\right)$ steadily built-up casbene during $96 \mathrm{~h}$ of cultivation where the measured casbene titer reached $61 \mathrm{mg} / \mathrm{L}$. This represented a significant $\sim 3$-fold improvement in production over the reference strain CAS2, which accumulated only $21 \mathrm{mg} / \mathrm{L}$ of casbene. At $120 \mathrm{~h}$ the casbene titer in CAS6 dropped to $\sim 40 \mathrm{mg} / \mathrm{L}$, indicating that potential degradation mechanisms or modifying enzymes start to act on the casbene molecule. Strain CAS8 $\left(\mathrm{P}_{E R G 1^{-}}\right.$ ERG20, $\left.\mathrm{P}_{E R G 1}-E R G 9\right)$ harboring dynamic control of expression of both ERG20 and ERG9 could reach the highest titer of $81.4 \mathrm{mg} / \mathrm{L}$ casbene after $96 \mathrm{~h}$ of cultivation, a $\sim 4$-fold improvement over production in CAS2. Strain CAS7 $\left(\mathrm{P}_{E R G 1^{-}}\right.$ ERG9) revealed instead the highest production after $48 \mathrm{~h}$ of cultivation $(42 \mathrm{mg} / \mathrm{L})$. Casbene titers steadily dropped to $18 \mathrm{mg} / \mathrm{L}$ thereafter. Finally, reference strain CAS2 (tHMGR) showed a gradual accumulation of casbene up $35 \mathrm{mg} / \mathrm{L}$ upon $72 \mathrm{~h}$ of culture, followed by a decline to $13.3 \mathrm{mg} / \mathrm{L}$ at $120 \mathrm{~h}$.

Also, GGOH considerably accumulated in the engineered strains (Figure 3B). Strains CAS7 ( $\left.\mathrm{P}_{E R G 1}-E R G 9\right)$ and CAS2 $(t H M G R)$ accumulated increasing amounts of GGOH during $72 \mathrm{~h}$ of cultivation, reaching up to $39.7 \mathrm{mg} / \mathrm{L}$, and $30.3 \mathrm{mg} / \mathrm{L}$, respectively. The titers of GGOH did not drop significantly throughout the entire cultivation period of $120 \mathrm{~h}$. Strains CAS6 ( $\left.\mathrm{P}_{E R G 1}-E R G 20\right)$ and CAS8 ( $\mathrm{P}_{E R G 1}-E R G 20, \mathrm{P}_{E R G 1}{ }^{-}$ ERG9) showed a progressive build-up of the compound reaching, upon $120 \mathrm{~h}$ of growth, $96.7 \mathrm{mg} / \mathrm{L}$ and $82.9 \mathrm{mg} / \mathrm{L}$, respectively. 
A

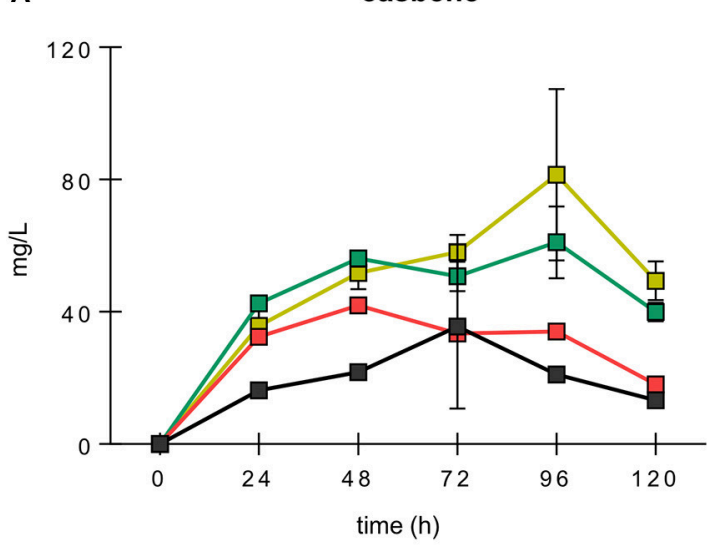

C

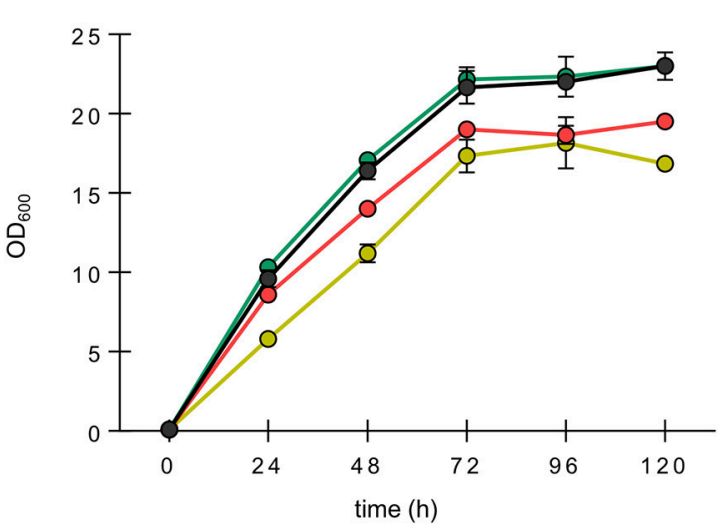

B

GGOH
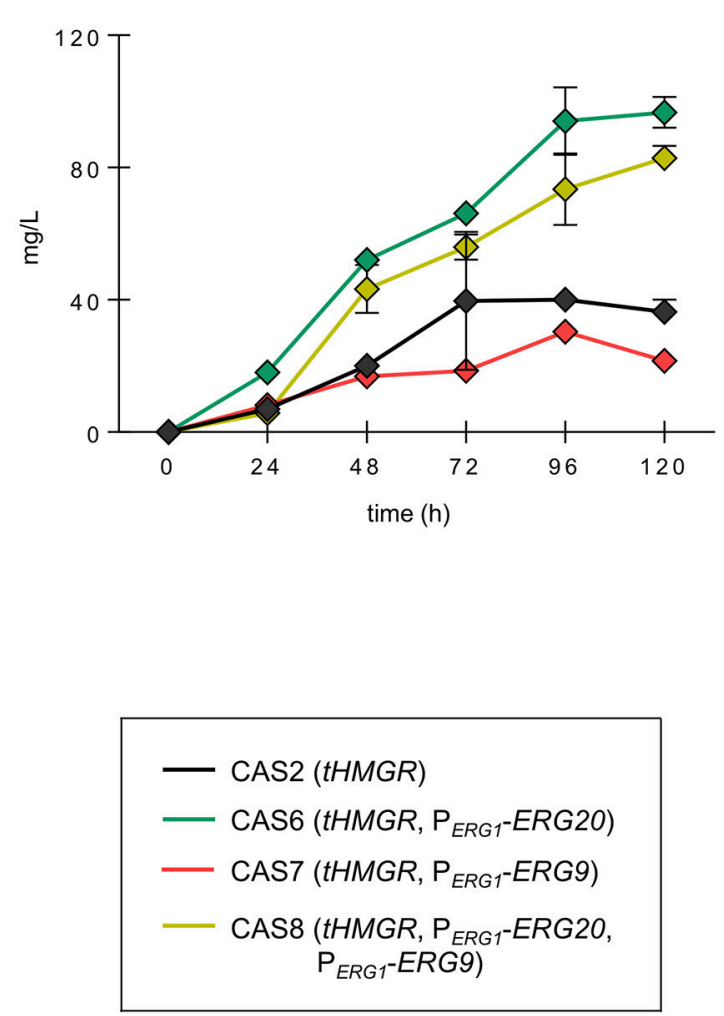

FIGURE 3 | Growth and production of casbene and GGOH in engineered strains expressing PaGGPPS and RcCBS. Time course of production of casbene (A) and GGOH (B) in strains CAS2 (tHMGR), CAS6 (tHMGR, $P_{E R G 1}$-ERG20), CAS7 (tHMGR, PERG1-ERG9), and CAS8 (tHMGR, PERG1-ERG20, PERG1-ERG9). All strains episomally expressed PaGGPPS and RCCBS. (C) Growth profile of the strains determined using optical density data recorded at $600 \mathrm{~nm}$ (OD 600 ). All strains were grown in selective medium for maximally $120 \mathrm{~h}$. Represented are the average and standard deviation of three independent experiments.

Strain CAS6 ( $\mathrm{P}_{E R G 1}-$ ERG20) in fact not only accumulated high amounts of both casbene and $\mathrm{GGOH}$, but also showed optimal robustness, as revealed by its growth profile, which was comparable to that of reference strain CAS2 (Figure 3C). Strains with dynamic control of ERG9 expression instead displayed growth retardation in comparison to reference strain CAS2. At the end of cultivation, strains CAS7 ( $\mathrm{P}_{E R G 1}$-ERG9) and CAS8 $\left(\mathrm{P}_{E R G 1}-E R G 20, \mathrm{P}_{E R G 1}-E R G 9\right)$ reached cell densities which were lower compared to strain CAS2 by 15 and $27 \%$, respectively.

These results demonstrated the potential of dynamically controlling FPP synthesis for improved casbene production via modification of the native ERG20 promoter.

\section{Construction of Stable S. cerevisiae Strains for Casbene Production}

To provide a stable chassis for casbene production, the engineered GGPP synthase PaGGPPS and the truncated casbene synthase RcCBS were stably integrated into the yeast chromosomes. In the previous plasmid-based assays, dynamic control of solely ERG9 expression showed the poorest performance in terms of production and was for this reason not included in the next phase of experiments. As dynamic control of ERG20 expression was shown to be crucial for improved diterpene production, we further investigated the effect of dynamic regulation of ERG20 by replacing its native promoter with the glucose-sensing promoter of HXT1 $\left(\mathrm{P}_{H X T 1}\right)$. It has recently been shown that using the HXT1 promoter to control ERG9 expression could redirect the carbon flux from sterol synthesis toward sesquiterpene production (Scalcinati et al., 2012). The effect of expression of ERG20 under control of $\mathrm{P}_{\text {HXT1 }}$ was tested either alone, or in combination with control of expression of ERG9 by $\mathrm{P}_{E R G 1}$ (Table 2, strains CAS9 and CAS10).

The expression cassettes for RcCBS and PaGGPPS were integrated into five strains: CAS11 (tHMGR), CAS12 (tHMGR, $\mathrm{P}_{\text {ERG1 }}$-ERG20), CAS13 (tHMGR, $\mathrm{P}_{\text {HXT1 }}$-ERG20), CAS14 (tHMGR, $\left.\mathrm{P}_{E R G 1}-E R G 20, \mathrm{P}_{E R G 1}-E R G 9\right)$ and CAS15 (tHMGR, 
$\left.\mathrm{P}_{H X T 1-E R G 20,} \mathrm{P}_{E R G 1}-E R G 9\right)$. All strains were analyzed for casbene and GGOH accumulation upon growth in batch culture for $96 \mathrm{~h}$, as this production time yielded the highest titers of casbene in plasmid-based assays.

All engineered strains accumulated higher amounts of casbene and GGOH compared to reference strain CAS11 (24.6 mg/L of casbene and $27.6 \mathrm{mg} / \mathrm{L}$ of $\mathrm{GGOH}$ ) (Figure 4). Strain CAS13 accumulated $85.5 \mathrm{mg} / \mathrm{L}$ of casbene and $50 \mathrm{mg} / \mathrm{L}$ of $\mathrm{GGOH}$, whereas strain CAS12 accumulated $66.5 \mathrm{mg} / \mathrm{L}$ of casbene and $63.4 \mathrm{mg} / \mathrm{L}$ of GGOH, respectively. In strain CAS14 (tHMGR, $\mathrm{P}_{E R G 1}$-ERG20, $\mathrm{P}_{E R G 1}$-ERG9) accumulation of casbene did not reach the amounts observed previously (only $49.1 \mathrm{mg} / \mathrm{L}$ of casbene and $46.5 \mathrm{mg} / \mathrm{L}$ of GGOH accumulated). In strain CAS15 expression of ERG20 controlled by $\mathrm{P}_{H X T 1}$ combined with control of ERG9 expression by promoter $\mathrm{P}_{E R G 1}$ led to the highest titers of casbene $(108.5 \mathrm{mg} / \mathrm{L})$ and GGOH $(79.9 \mathrm{mg} / \mathrm{L})$ production. Interestingly, all strains, except CAS14, grew better than the reference strain CAS11 ( $t H M G R$ only). CAS14 ( $t H M G R, \mathrm{P}_{E R G 1}$ $\left.E R G 20, \mathrm{P}_{E R G 1}-E R G 9\right)$ reached, in accordance with the plasmidbased expression results, lower final cell densities.

\section{DISCUSSION}

In this study we focused on the redirection of carbon flux toward casbene production by (1) introduction of a new biosynthetic branch for production of GGPP starting from IPP and DMAPP and (2) dynamic control of ERG20 and ERG9 expression by means of glucose- and ergosterol-sensitive promoters.

To increase the pool of GGPP in yeast, we expressed the GGPP synthase domain of the fusioccadiene synthase from $P$. amygdali. Similarly to other GGPP synthases from higher plants and bacteria (Vandermoten et al., 2009), this enzymatic domain synthesizes GGPP starting from one molecule of DMAPP and three molecules of IPP (Chen et al., 2016). In the yeast native pathway, GGPP is formed from the condensation of IPP and FPP, by action of the endogenous GGPP synthase Bts1p. FPP, in turn, is formed by the successive condensation of DMAPP with IPP units, catalyzed by FPP synthase, Erg20p. This requirement for two enzymatic steps for the production of GGPP, represents a limiting factor, partially due to the low native activity of Bts1p (Jiang et al., 1995). Low-titer diterpene production starting from the endogenous GGPP pool has been previously reported (Zhou et al., 2012; Ignea et al., 2015) and was confirmed in this study by the very low levels of casbene produced in yeast expressing RcCBS (Figure S1B). Improved efficiency of the conversion of FPP to GGPP was shown to significantly benefit diterpene production (Zhou et al., 2012; Ignea et al., 2015). Expression of $\mathrm{PaGGPPS}$ in yeast introduced the direct conversion of IPP and DMAPP to GGPP and improved the efficiency of GGPP formation. Indeed, expression of $P a G G P S+R c C B S$ lead to $32 \mathrm{mg} / \mathrm{L}$ casbene accumulation, a $\sim 40$ fold improvement compared to cells expressing solely $R c C B S$ and harnessing the endogenous source of GGPP. The substantial accumulation of GGOH in those cells clearly indicated the improved efficiency of GGPP biosynthesis and suggested that the flux toward diterpene synthesis could be further optimized.

To further increase the supply of IPP and DMAPP, we restricted the flux toward FPP and sterols, by limiting the activity of Erg20p and Erg9p. These two enzymes catalyze FPP synthesis and the first step in sterol biosynthesis, respectively. Sterols are essential for yeast growth, therefore it is crucial to ensure the delivery of intermediates and end-products at levels that can guarantee proper cell function. Ergosterol, the major product of sterol biosynthesis, fulfills in fact several vital cellular functions that require balanced sterol concentrations (Parks et al., 1995). Downregulation of Ergosterol synthesis by means of weak or inducible and repressible promoters has been shown to be beneficial for isoprenoid production, while negatively affecting yeast viability (Paradise et al., 2008; Fischer et al., 2011; Scalcinati et al., 2012; Ignea et al., 2014). Therefore, we engineered a dynamic control strategy to balance metabolism between diterpene formation and cell growth. $\mathrm{P}_{E R G 1}$ represents

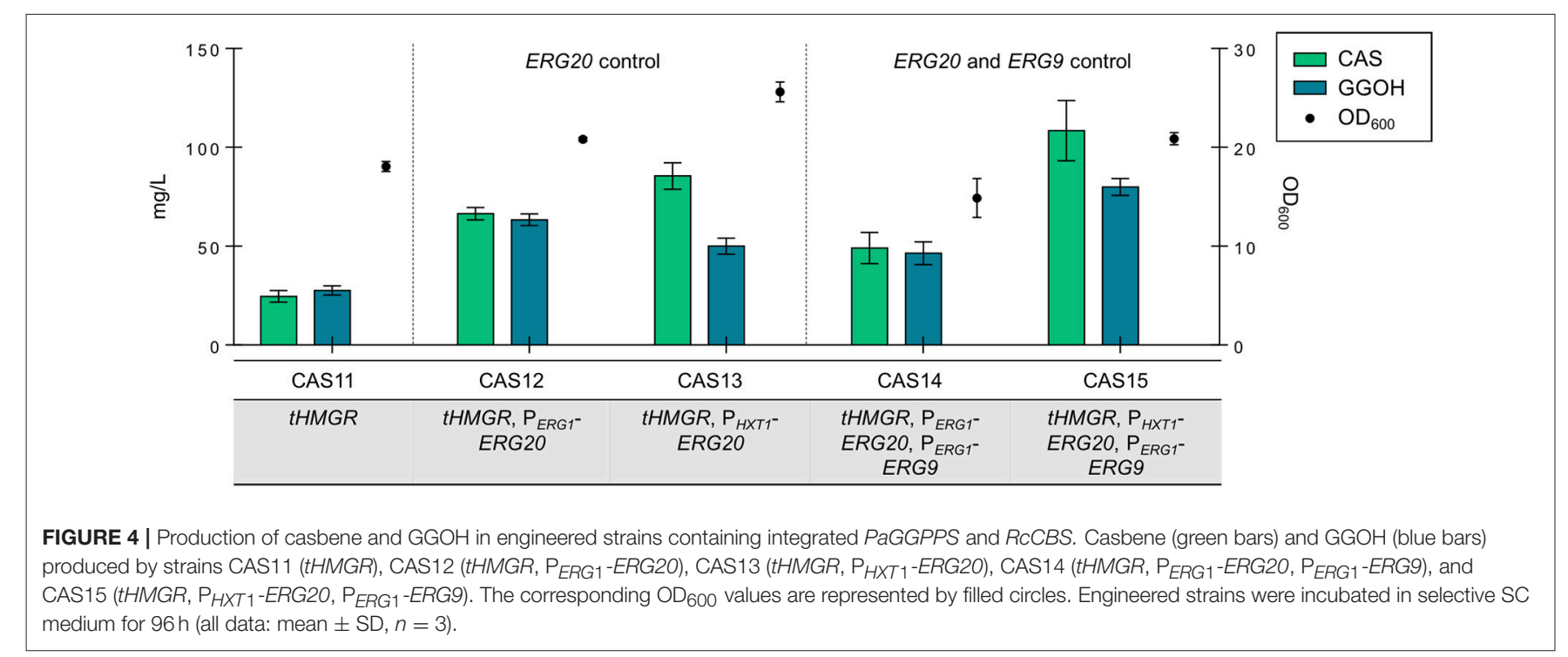


an ergosterol-sensitive promoter, previously shown to efficiently restrict ERG9 expression levels in order to boost amorphadiene production (FPP-derived) (Yuan and Ching, 2015). We applied a similar strategy to improve diterpene synthesis, extending dynamic control to ERG20, given the need to boost the IPP and DMAPP supply. Indeed, in our hands, the dynamic control of ERG9 alone did not have the remarkable improvement in sesquiterpene production that was reported by Yuan and Ching (2015). The strains harboring such a modification (tHMGR, $\mathrm{P}_{E R G 1}$-ERG9) produced amounts of casbene comparable to the reference strain $(t H M G R)$, at least in plasmid-based assays. Moreover, they accumulated the lowest titer of GGOH and reached lower cell densities. Enhancement of the mevalonate pathway (by $t H M G R$ overexpression), together with ERG9 down-regulation in the absence of a sesquiterpene synthase or another draining route, possibly led to accumulation of toxic concentrations of pathway intermediates, such as FPP. The toxicity of isoprenoid precursors (including FPP) has previously been reported in E. coli (Martin et al., 2003; Sarria et al., 2014).

Dynamic control of ERG20 by $\mathrm{P}_{E R G 1}$ showed instead to be a significant improvement, leading to a 3-fold increase in production, without negatively affecting growth. Titers reached even 4-fold higher values than the control strain when both ERG9 and ERG20 expression were controlled by $\mathrm{P}_{E R G 1}$. Downregulation of ERG20 alone, similar to the ERG9 downregulation reported by Yuan and Ching, most likely created a metabolic balance in which gene expression was adjusted to the cellular requirement for ergosterol biosynthesis. Once the cell sensed an excess of ergosterol, ERG20 expression was decreased, resulting in redirection of the metabolic flux from IPP/DMAPP to GGPP and non-native diterpenoids. The concerted downregulation of both ERG20 and ERG9, although leading to the highest casbene accumulation, might have resulted in an excessively restricted flux toward ergosterol which would explain the growth impairment observed upon dynamic control of both ERG9 and ERG20, leading to a $27 \%$ reduced cell density.

Integration of the expression constructs for $P a G G P P S$ and RcCBS in neutral loci of the yeast genome, together with dynamic control of ERG20 by means of the glucose-sensitive promoter of HXT1, further enhanced production of casbene. $\mathrm{P}_{\text {HXT1 }}$ allows for moderate expression levels when glucose is present in the medium, but leads to gene repression when glucose concentration is low or absent. This promoter was previously used for dynamic control of ERG20 expression in strains engineered for improved production of geraniol (Zhao et al., 2017). When used for dynamic control of ERG20, $\mathrm{P}_{\text {HXT1 }}$ had a beneficial effect on both production and growth compared to $\mathrm{P}_{E R G 1}$. Repression of expression by $\mathrm{P}_{H X T 1}$ might be stronger than the one imposed by $\mathrm{P}_{E R G 1}$, leading to a higher supply of DMAPP and IPP for the heterologous GGPP synthase, thus explaining the higher titers. Moreover, since repression of expression mediated by $\mathrm{P}_{\text {HXT1 }}$ occurs when glucose concentration is low, its control possibly maximized the carbon flux to ergosterol and biomass, thus explaining the higher cell densities. Combining dynamic control of ERG20 by $\mathrm{P}_{\text {HXT1 }}$ with dynamic control of ERG9 by $\mathrm{P}_{E R G 1}$ led to the highest casbene titer of $108.5 \mathrm{mg} / \mathrm{L}$ and worked best to guarantee a proper flux distribution between cell growth and diterpene synthesis.

Proper adjustment and usage of carbon source sensitive promoters, to improve casbene or in general diterpenoid production, opens up new perspectives for an extensive optimization of the culturing conditions during fermentation that were beyond the scope of this study. For example, substantial improvements in isoprenoid biosynthesis have been reported when ethanol is provided as the carbon source (Westfall et al., 2012; Zhao et al., 2017). In the future, it would therefore be interesting to analyze the effect of combining feeding with ethanol or with mixture of different carbon sources with ERG20 expression under control of $\mathrm{P}_{H X T 1}$ for casbene production.

Overall these results showed remarkable improvements in diterpene production via an engineered heterologous route toward synthesis of GGPP, combined with dynamic regulation of competing pathways. However, a substantial accumulation of GGOH could still be observed in all our engineered strains, indicating that not all of the GGPP produced is channeled toward casbene biosynthesis. This may be due to the low efficiency of the casbene synthase which, similarly to other enzymes of the secondary metabolism of plants, has not evolved to synthesize high titers of products. During preparation of this manuscript, a casbene production of 160 $\mathrm{mg} / \mathrm{L}$ was reported in a yeast strain expressing multiple copies of casbene synthase constructs, with improved solubility achieved by protein tagging strategies (Wong et al., 2017). Titers in our strains could most likely similarly be improved by increasing the copy number of the casbene synthase gene. Moreover, fusion between the GGPP synthase PaGGPPS and the casbene synthase RcCBS could possibly offer an enzyme with improved solubility and a more efficient substrate channeling that mimics the natural chimera fusicoccadiene synthase. Expression of such a chimera may further increase production levels.

In conclusion, although further optimization is needed, the design developed here provides a valuable strategy for sustainable production of casbene derived chemicals. Moreover, the approach can easily be adapted to synthesis of other GGPPderived compounds.

\section{AUTHOR CONTRIBUTIONS}

RC designed experiments, performed strain constructions, extractions, analysis of samples, and drafted the manuscript. DR established the analysis methods. YM helped in construction of the integration strains. $\mathrm{RC}$ and $\mathrm{HH}$ coordinated the study. $\mathrm{HH}$ reviewed and edited the manuscript. All authors read and approved the final manuscript.

\section{FUNDING}

This work was supported by the Danish Innovation Foundation funded project Plant Power: light-driven synthesis of complex terpenoids using cytochrome P450s (12-131834; project lead, Dr. Poul Erik Jensen, University of Copenhagen). 


\section{ACKNOWLEDGMENTS}

We thank Christophe Folly for support in initial GC-MS analysis, Michael Eichenberger for providing plasmid pCAS4, Dr Samantha Capewell for proofreading the manuscript and Professor Birger Møller for continuous support and for supplying the casbene standard.

\section{REFERENCES}

Bohlmann, J., and Keeling, C. I. (2008). Terpenoid biomaterials. Plant J. 54, 656-669. doi: 10.1111/j.1365-313X.2008.03449.x

Callari, R. (2018). Pathway Engineering Towards Heterologous Production of Complex Diterpenoids in Yeast. PhD thesis. Department of Plant and Environmental Sciences, Faculty of Science, University of Copenhagen.

Caniard, A., Zerbe, P., Legrand, S., Cohade, A., Valot, N., Magnard, J. L., et al. (2012). Discovery and functional characterization of two diterpene synthases for sclareol biosynthesis in Salvia sclarea (L.) and their relevance for perfume manufacture. BMC Plant Biol. 12:119. doi: 10.1186/1471-2229-12-119

Chambon, C., Ladeveze, V., Servouse, M., Blanchard, L., Javelot, C., Vladescu, B., et al. (1991). Sterol pathway in yeast. Identification and properties of mutant strains defective in mevalonate diphosphate decarboxylase and farnesyl diphosphate synthetase. Lipids 26, 633-636. doi: 10.1007/BF02536428

Chen, M., Chou, W. K., Toyomasu, T., Cane, D. E., and Christianson, D. W. (2016). Structure and Function of Fusicoccadiene Synthase, a Hexameric Bifunctional Diterpene Synthase. ACS Chem. Biol. 11, 889-899. doi: 10.1021/acschembio.5b00960

Cheng, A.-X., Lou, Y.-G., Mao, Y.-B., Lu, S., Wang, L.-J., and Chen, X.-Y. (2007). Plant terpenoids: biosynthesis and ecological functions. J. Integr. Plant Biol. 49, 179-186. doi: 10.1111/j.1744-7909.2007.00395.x

Donald, K. A., Hampton, R. Y., and Fritz, I. B. (1997). Effects of overproduction of the catalytic domain of 3-hydroxy-3-methylglutaryl coenzyme A reductase on squalene synthesis in Saccharomyces cerevisiae. Appl. Environ. Microbiol. 63, 3341-3344.

Doseyici, S., Mehmetoglu, I., Toker, A., Yerlikaya, F. H., and Erbay, E. (2014). The effects of forskolin and rolipram on cAMP, cGMP and free fatty acid levels in diet induced obesity. Biotech. Histochem. 89, 388-392. doi: 10.3109/10520295.2014.883463

Emanuelsson, O., Nielsen, H., and von Heijne, G. (1999). ChloroP, a neural network-based method for predicting chloroplast transit peptides and their cleavage sites. Protein Sci. 8, 978-984. doi: 10.1110/ps.8.5.978

Faulkner, A., Chen, X., Rush, J., Horazdovsky, B., Waechter, C. J., Carman, G. M., et al. (1999). The LPP1 and DPP1 gene products account for most of the isoprenoid phosphate phosphatase activities in Saccharomyces cerevisiae. J. Biol. Chem. 274, 14831-14837. doi: 10.1074/jbc.274.21.14831

Fidler, B., and Goldberg, T. (2014). Ingenol mebutate gel (picato): a novel agent for the treatment of actinic keratoses. PT 39, 40-46.

Fischer, M. J., Meyer, S., Claudel, P., Bergdoll, M., and Karst, F. (2011). Metabolic engineering of monoterpene synthesis in yeast. Biotechnol. Bioeng. 108, 1883-1892. doi: 10.1002/bit.23129

Gietz, R. D., and Schiestl, R. H. (2007). High-efficiency yeast transformation using the LiAc/SS carrier DNA/PEG method. Nat. Protoc. 2, 31-34. doi: $10.1038 /$ nprot.2007.13

Goyal, S. K., Samsher, and Goyal, R. K. (2010). Stevia (Stevia rebaudiana) a bio-sweetener: a review. Int. J. Food Sci. Nutr. 61, 1-10. doi: 10.3109/09637480903193049

Green, M. R., and Sambrook, J. (2012). Molecular Cloning: A Laboratory Manual: Three-Volume Set, 4th edn. Cold Spring Harbor Laboratory Press.

Gueldener, U., Heinisch, J., Koehler, G. J., Voss, D., and Hegemann, J. H. (2002). A second set of loxP marker cassettes for Cre-mediated multiple gene knockouts in budding yeast. Nucleic Acids Res. 30:e23. doi: 10.1093/nar/30.6.e23

Hamann, T., and Møller, B. L. (2007). Improved cloning and expression of cytochrome P450s and cytochrome P450 reductase in yeast. Protein Expr. Purif. 56, 121-127. doi: 10.1016/j.pep.2007.06.007
The content of this research was first reported and is adapted from the PhD thesis of RC (Callari, 2018).

\section{SUPPLEMENTARY MATERIAL}

The Supplementary Material for this article can be found online at: https://www.frontiersin.org/articles/10.3389/fbioe. 2018.00160/full\#supplementary-material

Howat, S., Park, B., Oh, I. S., Jin, Y. W., Lee, E. K., and Loake, G. J. (2014). Paclitaxel: biosynthesis, production and future prospects. N. Biotechnol. 31, 242-245. doi: 10.1016/j.nbt.2014.02.010

Iadarola, M. J., and Gonnella, G. L. (2013). Resiniferatoxin for pain treatment: an interventional approach to personalized pain medicine. Open Pain J. 6, 95-107. doi: 10.2174/1876386301306010095

Ignea, C., Pontini, M., Maffei, M. E., Makris, A. M., and Kampranis, S. C. (2014). Engineering monoterpene production in yeast using a synthetic dominant negative geranyl diphosphate synthase. ACS Synth. Biol. 3, 298-306. doi: $10.1021 / \mathrm{sb} 400115 \mathrm{e}$

Ignea, C., Trikka, F. A., Nikolaidis, A. K., Georgantea, P., Ioannou, E., Loupassaki, S., et al. (2015). Efficient diterpene production in yeast by engineering Erg20p into a geranylgeranyl diphosphate synthase. Metab. Eng. 27, 65-75. doi: 10.1016/j.ymben.2014.10.008

Jiang, Y., Proteau, P., Poulter, D., and Ferro-Novick, S. (1995). BTS1 encodes a geranylgeranyl diphosphate synthase in Saccharomyces cerevisiae. J. Biol. Chem. 270, 21793-21799. doi: 10.1074/jbc.270.37.21793

Kirby, J., and Keasling, J. D. (2009). Biosynthesis of plant isoprenoids: perspectives for microbial engineering. Annu. Rev. Plant Biol. 60, 335-355. doi: 10.1146/annurev.arplant.043008.091955

Kirby, J., Nishimoto, M., Park, J. G., Withers, S. T., Nowroozi, F., Behrendt, D., et al. (2010). Cloning of casbene and neocembrene synthases from Euphorbiaceae plants and expression in Saccharomyces cerevisiae. Phytochemistry 71, 1466-1473. doi: 10.1016/j.phytochem.2010.06.001

Kupchan, S. M., Sigel, C. W., Matz, M. J., Saenz Renauld, J. A., Haltiwanger, R. C., and Bryan, R. F. (1970). Jatrophone, a novel macrocyclic diterpenoid tumor inhibitor from Jatropha gossypiifolia. J. Am. Chem. Soc. 92, 4476-4477. doi: 10.1021/ja00717a066

Lin, M., Tang, S., Zhang, C., Chen, H., Huang, W., Liu, Y., et al. (2017). Euphorbia factor L2 induces apoptosis in A549 cells through the mitochondrial pathway. Acta Pharm. Sin B 7, 59-64. doi: 10.1016/j.apsb.2016.06.008

Martin, V. J., Pitera, D. J., Withers, S. T., Newman, J. D., and Keasling, J. D. (2003). Engineering a mevalonate pathway in Escherichia coli for production of terpenoids. Nat. Biotechnol. 21, 796-802. doi: 10.1038/nbt833

Mau, C. J., and West, C. A. (1994). Cloning of casbene synthase cDNA: evidence for conserved structural features among terpenoid cyclases in plants. Proc. Natl. Acad. Sci. U.S.A. 91, 8497-8501. doi: 10.1073/pnas.91.18.8497

Miana, G. A., Riaz, M., Shahzad-ul-Hussan, S., Paracha, R. Z., and Paracha, U. Z. (2015). Prostratin: an overview. Mini Rev. Med. Chem. 15, 1122-1130. doi: 10.2174/1389557515666150511154108

Paradise, E. M., Kirby, J., Chan, R., and Keasling, J. D. (2008). Redirection of flux through the FPP branch-point in Saccharomyces cerevisiae by down-regulating squalene synthase. Biotechnol. Bioeng. 100, 371-378. doi: 10.1002/bit.21766

Parks, L. W., Smith, S. J., and Crowley, J. H. (1995). Biochemical and physiological effects of sterol alterations in yeast-A review. Lipids 30, 227-230. doi: 10.1007/BF02537825

Sarria, S., Wong, B., García Martín, H., Keasling, J. D., and Peralta-Yahya, P. (2014). Microbial synthesis of pinene. ACS Synth. Biol. 3, 466-475. doi: $10.1021 /$ sb4001382

Scalcinati, G., Knuf, C., Partow, S., Chen, Y., Maury, J., Schalk, M., et al. (2012). Dynamic control of gene expression in Saccharomyces cerevisiae engineered for the production of plant sesquitepene $\alpha$-santalene in a fed-batch mode. Metab. Eng. 14, 91-103. doi: 10.1016/j.ymben.2012.01.007

Song, T. Q., Ding, M. Z., Zhai, F., Liu, D., Liu, H., Xiao, W. H., et al. (2017). Engineering Saccharomyces cerevisiae for geranylgeraniol overproduction by combinatorial design. Sci. Rep. 7:14991. doi: 10.1038/s41598-017-15005-4 
Theoduloz, C., Rodríguez, J. A., Pertino, M., and Schmeda-Hirschmann, G. (2009). Antiproliferative activity of the diterpenes jatrophone and jatropholone and their derivatives. Planta Med. 75, 1520-1522. doi: 10.1055/s-0029-1185834

Tholl, D. (2015). Biosynthesis and biological functions of terpenoids in plants. $A d v$. Biochem. Eng. Biotechnol. 148, 63-106. doi: 10.1007/10_2014_295

Tokuhiro, K., Muramatsu, M., Ohto, C., Kawaguchi, T., Obata, S., Muramoto, N., et al. (2009). Overproduction of geranylgeraniol by metabolically engineered Saccharomyces cerevisiae. Appl. Environ. Microbiol. 75, 5536-5543. doi: 10.1128/AEM.00277-09

Toyomasu, T., Tsukahara, M., Kaneko, A., Niida, R., Mitsuhashi, W., Dairi, T., et al. (2007). Fusicoccins are biosynthesized by an unusual chimera diterpene synthase in fungi. Proc. Natl. Acad. Sci. U.S.A. 104, 3084-3088. doi: 10.1073/pnas.0608426104

Vandermoten, S., Haubruge, E., and Cusson, M. (2009). New insights into short-chain prenyltransferases: structural features, evolutionary history and potential for selective inhibition. Cell. Mol. Life Sci. 66, 3685-3695. doi: 10.1007/s00018-009-0100-9

Westfall, P. J., Pitera, D. J., Lenihan, J. R., Eng, D., Woolard, F. X., Regentin, R., et al. (2012). Production of amorphadiene in yeast, and its conversion to dihydroartemisinic acid, precursor to the antimalarial agent artemisinin. Proc. Natl. Acad. Sci. U.S.A. 109, E111-E118. doi: 10.1073/pnas.1110 740109

Wong, J., de Rond, T., d'Espaux, L., van der Horst, C., Dev, I., Rios-Solis, L., et al. (2017). High-titer production of lathyrane diterpenoids from sugar by engineered Saccharomyces cerevisiae. Metab. Eng. 45, 142-148. doi: 10.1016/j.ymben.2017.12.007
Yuan, J., and Ching, C. B. (2015). Dynamic control of ERG9 expression for improved amorpha-4,11-diene production in Saccharomyces cerevisiae. Microb. Cell Fact. 14:38. doi: 10.1186/s12934-015-0220-x

Zerbe, P., and Bohlmann, J. (2015). Enzymes for synthetic biology of ambroxiderelated diterpenoid fragrance compounds. Adv. Biochem. Eng. Biotechnol. 148, 427-447. doi: 10.1007/10_2015_308

Zhao, J., Li, C., Zhang, Y., Shen, Y., Hou, J., and Bao, X. (2017). Dynamic control of ERG20 expression combined with minimized endogenous downstream metabolism contributes to the improvement of geraniol production in Saccharomyces cerevisiae. Microb. Cell Fact. 16:17. doi: 10.1186/s12934-017-0641-9

Zhou, Y. J., Gao, W., Rong, Q., Jin, G., Chu, H., Liu, W., et al. (2012). Modular pathway engineering of diterpenoid synthases and the mevalonic acid pathway for miltiradiene production. J. Am. Chem. Soc. 134, 3234-3241. doi: $10.1021 /$ ja2 114486

Conflict of Interest Statement: All authors were or are employees of the company Evolva SA. Evolva SA is listed on the Swiss stock exchange.

Copyright (c) 2018 Callari, Meier, Ravasio and Heider. This is an open-access article distributed under the terms of the Creative Commons Attribution License (CC BY). The use, distribution or reproduction in other forums is permitted, provided the original author(s) and the copyright owner(s) are credited and that the original publication in this journal is cited, in accordance with accepted academic practice. No use, distribution or reproduction is permitted which does not comply with these terms. 